\title{
Primary extracranial meningioma presenting as a forehead mass
}

\author{
Chae Min Kim \\ Yeo Reum Jeon ${ }^{1}$, \\ Yee Jeong Kim², \\ Seum Chung ${ }^{1}$ \\ Departments of ${ }^{1}$ Plastic and Reconstructive \\ Surgery and ${ }^{2}$ Pathology, National Health \\ Insurance Service Ilsan Hospital, Ilsan, Korea
}

\begin{abstract}
Meningioma originates from arachnoid cap cells and is the second most common intracranial tumor; however, it can also be found in an extracranial location. A very rare primary extracranial meningioma without the presence of an intracranial component has also been reported. Primary extracranial meningiomas have been found in the skin, scalp, middle ear, and nasal cavity. A computerized tomography or magnetic resonance imaging scan is necessary to determine the presence or absence of an intracranial meningioma, and a biopsy is essential for diagnosis. We report a case of primary extracranial meningioma located in the forehead skin of a 51-year-old male.
\end{abstract}

Keywords: Meningioma / Forehead / Subcutaneous tissue

\section{INTRODUCTION}

Meningioma accounts for approximately $20 \%-30 \%$ of all intracranial neoplasms and is the second most common neoplasm of the central nervous system [1,2]. Meningiomas mainly occur in middle-aged women, and it is reported that women are almost twice as likely as men to develop intracranial meningiomas and five to 10 times more likely to develop spinal meningiomas. Most meningiomas are benign (World Health Organization [WHO] grade I), but $20 \%$ are atypical (WHO grade II), and $1 \%$ to $3 \%$ are so-called anaplastic (malignant) meningiomas [3]. Rarely, meningiomas can be found in the extradural region. These extracranial meningiomas can be divided into primary extracranial meningioma and secondary extracranial meningioma depending on the presence of an intracranial tumor component [4]. Among them, primary extracranial meningioma with no intracranial component is extremely rare (0.9\%) [5]. These primary extracranial meningiomas are likely to be misdiagnosed as other tumors due to

\section{Correspondence: Seum Chung}

Department of Plastic and Reconstructive Surgery, National Health Insurance Service Ilsan Hospital, 100 Ilsan-ro, Ilsandong-gu, Goyang 10444, Korea

E-mail: schung@nhimc.or.kr

Received October 12, 2017 / Revised January 23, 2018 / Accepted February 7, 2018 the absence of characteristic symptoms. We present a case of histologically confirmed primary extracranial meningioma of the forehead skin.

\section{CASE REPORT}

A 51-year-old male had a symptomless forehead mass of about 1-year duration. Excision was attempted at a private clinic but was discontinued due to excessive bleeding. At the time of presentation at National Health Insurance Service Ilsan Hospital (Ilsan,

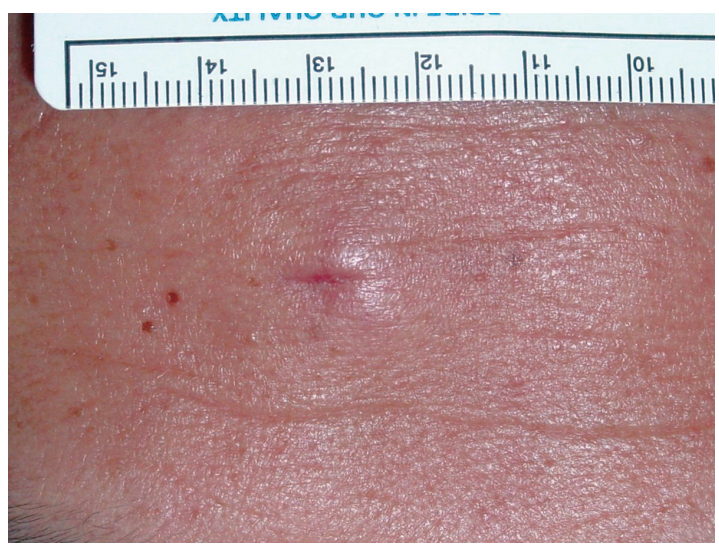

Fig. 1. Protruding mass and scar on the forehead. 
Korea), there was an approximately $1.5-\mathrm{cm}$-diameter firm protruding mass with an overlying $0.5-\mathrm{cm}$-long incision scar on the middle forehead (Fig. 1). An ultrasonogram showed a 2.05×1.36× 0.49-cm benign-looking hyperechoic nodular lesion (Fig. 2). A subsequent computerized tomography (CT) scan showed that the lesion was confined to the extracranial location (Fig. 3). An excisional biopsy was performed under local anesthesia. During the excisional biopsy, the mass was difficult to grossly delineate from the overlying skin and was found to be adhered to the underlying frontalis muscle. Therefore, the mass was excised en-bloc in an elliptical shape including the skin and frontalis muscle. The wound was closed by primary repair. Histopathology showed a lobular architecture composed of tumor cells with eosinophilic cytoplasm and indistinct cell borders (Fig. 4). A few nuclear pseudoinclusions were also noted (Fig. 5). Diffuse granular cytoplasmic immunostaining was positive for epithelial membrane antigen (Fig. 6). These findings were consistent with an extracranial meningioma. A brain computed tomography scan showed no re- markable findings in the intracranial structures. Therefore, a final diagnosis of primary extracranial meningioma was made. Currently, there is no evidence of recurrence at 3 years after surgery.

\section{DISCUSSION}

Extracranial meningiomas are classified according to Hoye et al. [4]. Primary extracranial meningioma and secondary extracranial meningioma can be classified according to the presence or absence of an intracranial meningioma. Primary extracranial meningiomas are composed of two types: a tumor originating from arachnoid cells remaining in the nerve sheaths and a tumor occurring ectopically without connection to the cranium. Secondary extracranial meningiomas are classified as intracranial tumors directly escaping from the cranium and extracranial meningioma that has metastasized from an intracranial meningioma [6]. According to this classification, our case is classified as an ectopic primary extracranial meningioma.

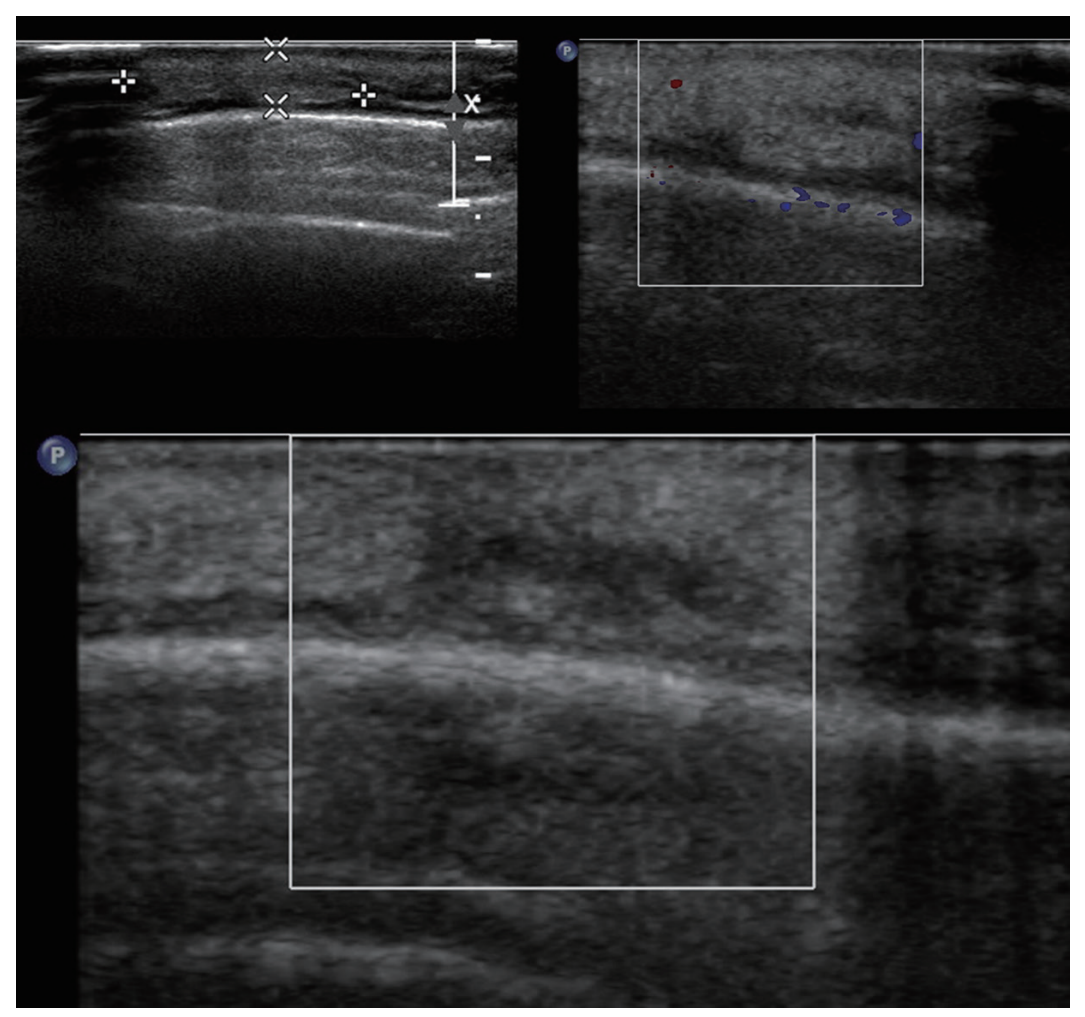

Fig. 2. Ultrasonographic findings showed a $2.05 \times 1.36 \times 0.49-\mathrm{cm}$ benign-looking hyperechoic nodular lesion on forehead. There were no abnormal vascular flow. 


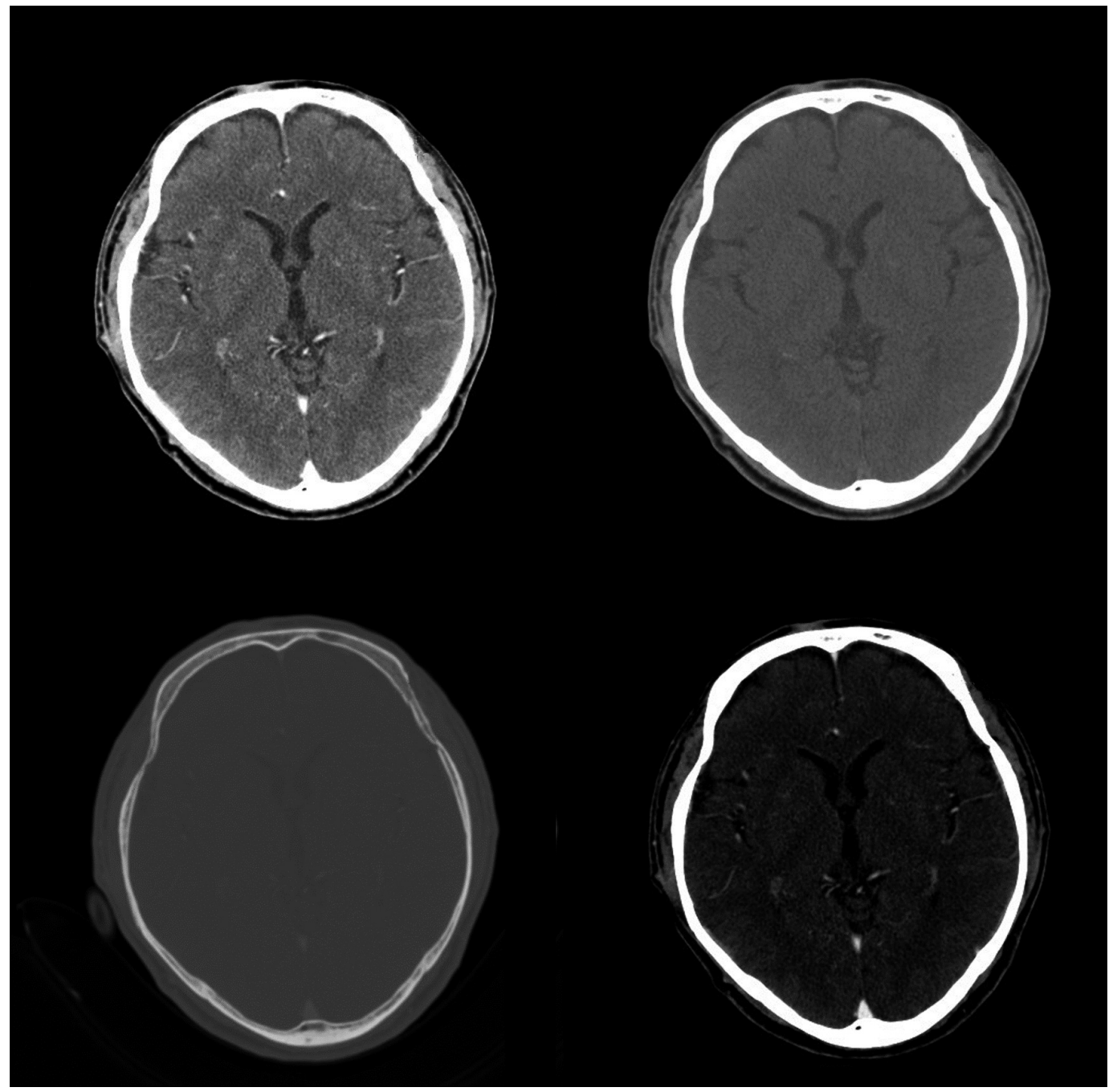

Fig. 3. Brain computerized tomography scan with various windows showed a tiny hyperdense lesion adjacent to the frontal bone. There was no other remarkable finding in the intracranial structures.

Intradural meningioma is known to be associated with radiation, virus, head trauma, smoking, nitrate, and high cholesterol levels, but the cause of primary extracranial meningioma is not yet clear. Possible hypotheses are as follows: (1) migration of arachnoid cells along the cranial nerve sheath; (2) proliferation of extradural arachnoid cells that have become disarranged during embryogenesis; (3) displacement of arachnoid islets by trauma or cerebral hypertension; (4) origination from pluripotent mesenchymal cells.

Rushing et al. [1] examined 146 cases of primary extracranial meningiomas. The ratio of males to females was 74 to 72 , showing similar incidence between sexes. The most common location was skin and scalp (40.4\%), followed by middle ear (17.8\%), and nasal cavity (11.6\%). Tumor size ranged from $0.5 \mathrm{~cm}$ to $4.5 \mathrm{~cm}$, with an average of $1.2 \mathrm{~cm}$. Extracranial meningioma usually shows a simple polyp-like appearance of a firm to rubbery mass, and it is not easy to distinguish from other masses [7]. Therefore, different types of benign or malignant tumors should be differentiated according to anatomical location.

It is also difficult to diagnose extracranial meningioma on CT scan. It usually appears as a homogeneous enhancing mass, and calcification can occur. A magnetic resonance imaging scan 


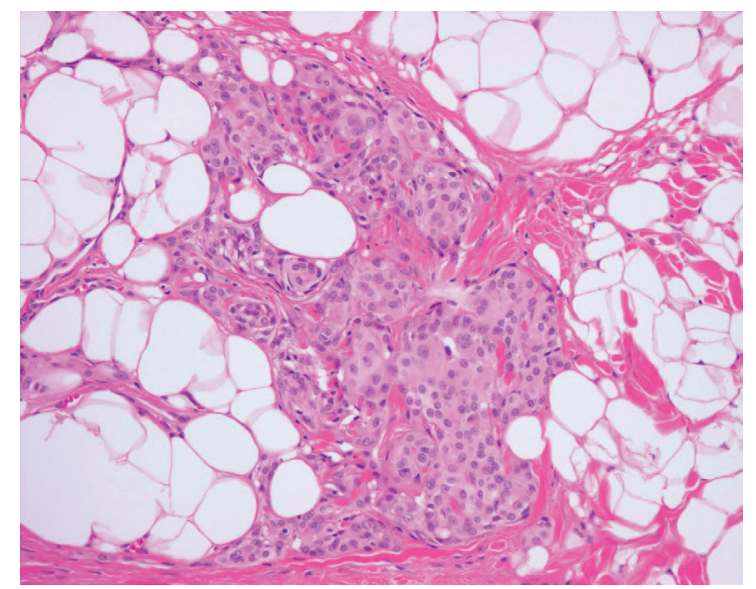

Fig. 4. Lobular architecture composed of tumor cells with eosinophilic cytoplasm and indistinct cell border $(\mathrm{H} \& \mathrm{E}, \times 200)$.

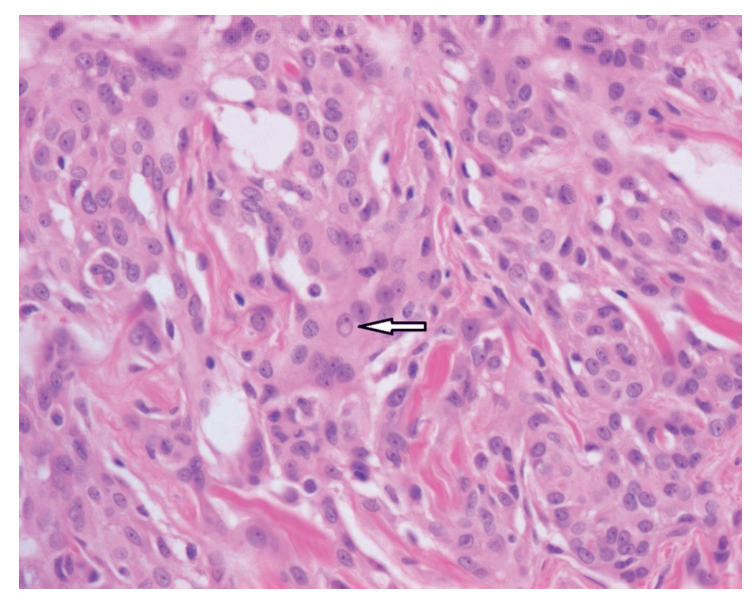

Fig. 5. A few nuclear pseudoinclusions (arrow) (H\&E, $\times 400)$.

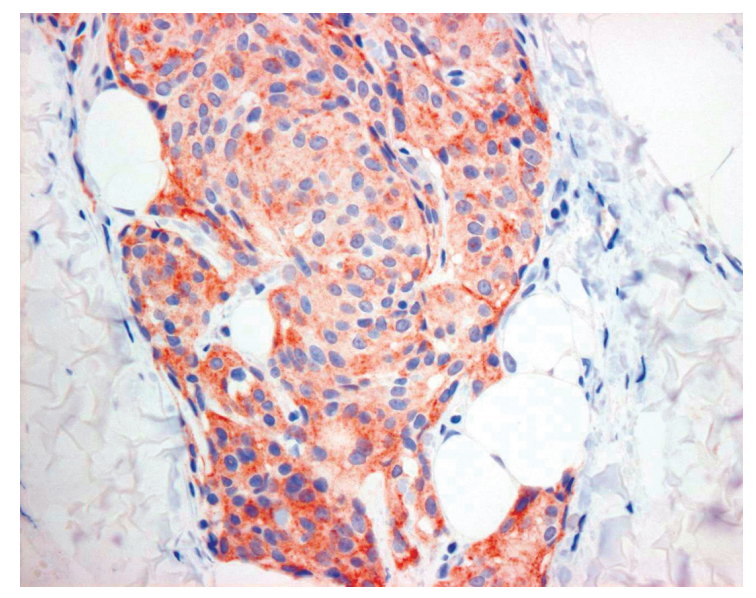

Fig. 6. Diffuse granular cytoplasmic immunostaining for epithelial membrane antigen $(\times 400)$. shows a homogeneous mass with similar signal intensity to the brain parenchyma [8]. Vascular flow is often not visible on angiography [9].

Therefore, the final diagnosis is confirmed by pathology. Meningiomas have various pathologic findings, including meningiothelial (syncytial), fibrous, psammomatous, angiomatous, and transitional meningioma [3]. Our patient showed a meningiothelial pattern. The mass was composed of uniform tumor cells forming lobules, surrounded by collagenous septae. Ill-defined cell borders were seen within the lobules. It also showed a characteristic nuclear change, including a clear space called the pseudoinclusion. Immunohistochemistry is essential for the diagnosis of extracranial meningioma. Meningiomas are positive for epithelial membrane antigen and vimentin and can be positive for S-100 protein, cytokeratin, and desmin [10]. These results were all seen in our patient.

Extracranial meningioma is a rare tumor of the head and neck. If extracranial meningioma is diagnosed pathologically, it is important to confirm the presence of an intracranial component by radiological examination. Treatment of choice for extracranial meningioma is complete surgical resection. Recurrence after complete resection is rare. [10,11].

\section{CONFLICT OF INTEREST}

No potential conflict of interest relevant to this article was reported.

\section{PATIENT CONSENT}

The patients provided written informed consent for the publication and the use of their images.

\section{REFERENCES}

1. Rushing EJ, Bouffard JP, McCall S, Olsen C, Mena H, Sandberg GD, et al. Primary extracranial meningiomas: an analysis of 146 cases. Head Neck Pathol 2009;3:116-30.

2. Wadhera R, Hernot S, Kaintura M, Bhukar S, Dheeraj S. Primary extracranial meningioma as a very rare cause of nasal mass and epistaxis in an elderly. J Clin Diagn Res 2016;10:MD01-03. 
3. Riemenschneider MJ, Perry A, Reifenberger G. Histological classification and molecular genetics of meningiomas. Lancet Neurol 2006;5:1045-54.

4. Hoye SJ, Hoar CS Jr, Murray JE. Extracranial meningioma presenting as a tumor of the neck. Am J Surg 1960;100:486-9.

5. Batsakis JG. Pathology consultation: extracranial meningiomas. Ann Otol Rhinol Laryngol 1984;93(3 Pt 1):282-3.

6. Willen R, Gad A, Willen H, Qvarnstrom O, Stahle J. Extracranial meningioma presenting as a nasal polyp. ORL J Otorhinolaryngol Relat Spec 1979;41:234-9.

7. Aiyer RG, Prashanth V, Ambani K, Bhat VS, Soni GB. Primary extracranial meningioma of paranasal sinuses. Indian J Otolaryngol Head
Neck Surg 2013;65(Suppl 2):384-7.

8. Lee DH, Sim HS, Hwang JH, Kim KS, Lee SY. Extracranial meningioma presenting as an eyebrow mass. J Craniofac Surg 2017;28:e305-7.

9. Shetty C, Avinash KR, Auluck A, Mupparapu M. Extracranial meningioma of the parapharyngeal space: report of a case and review of the literature. Dentomaxillofac Radiol 2007;36:117-20.

10. Maeng JW, Kim YH, Seo J, Kim SW. Primary extracranial meningioma presenting as a cheek mass. Clin Exp Otorhinolaryngol 2013;6:266-8.

11. Iaconetta G, Santella A, Friscia M, Abbate V, Califano L. Extracranial primary and secondary meningiomas. Int J Oral Maxillofac Surg 2012;41:211-7. 